\title{
COMPARACIÓN IN VITRO DEL CAMBIO DE MICRODUREZA SUPERFICIAL DENTINARIA ENTRE DOS SECUENCIAS DE IRRIGACIÓN ENDODÓNTICA
}

\section{IN VITRO COMPARISON OF DENTINAL SUPERFICIAL MICRO- HARDNESS CHANGE BETWEEN TWO ENDODONTIC IRRIGATION SEQUENCES}

Irigoyen Aitziber ${ }^{1}$, Santis Sofía², Soto Iván², Marchant Carlos ${ }^{4}$

\section{RESUMEN}

Objetivo: Comparar el cambio de microdureza superficial de la dentina radicular (MSD) ante dos secuencias de irrigación (SI) mediante prueba de microdureza Vickers (VHN).

Materiales y Métodos: 10 dientes humanos unirradiculados se cortaron longitudinalmente para obtener 20 muestras. Se prepararon dos SI, una tradicional $(\mathrm{NaOCl} 5 \%$, suero $0,9 \%$, EDTA $17 \%$, suero $0.9 \%$ ) y una modificada (tradicional más irrigación final con $\mathrm{NaOCl} 5 \%$ + suero $0,9 \%$ ), separando cada irrigante en una placa de vidrio. En dos grupos de 10 muestras (SI tradicional y SI modificada) se realizaron 5 identaciones con microdurómetro Vickers antes y después de sumergir las muestras en las SI. Se obtuvo el promedio de microdureza Vickers (VHN) de las muestras y se compararon estadísticamente. $\mathrm{P}$ valor de 0.05 .

Resultados: Se obtuvieron diferencias estadísticamente significativas entre la microdureza superficial dentinaria medida pre-irrigación (control) y post-irrigación en ambos protocolos, sin embargo, no se encontraron diferencias estadísticamente significativas entre ambos protocolos.

Conclusión: Las SI -tanto tradicional como modificada generan los mismos cambios en la microdureza superficial de la dentina radicular no presentando diferencias entre una y otra.
1. Centro de Salud Familiar Cordillera, Valparaíso, Chile.

2. Atención Privada Odontológica, San Antonio, Chile.

4. Universidad de Valparaíso, Escuela de Odontología, Valparaíso, Chile.

Correspondencia:

Carlos Marchant Pizzarro. Escuela de Odontología. Subida Leopoldo Carvallo \#211, Valparaíso, Chile.

Correo electrónico:

carlos.marchant@uv.cl

\section{PALABRAS CLAVES:}

Endodoncia, Irrigantes, Sellado Coronal, Sistemas Adhesivos, Capa híbrida.

\section{KEYWORDS:}

Endodontics, irrigators, coronal sealing, adhesive systems, hybrid layer. 


\section{ABSTRACT}

Objectives: to compare changes in surface microhardness of dentinal root (MSD) with two irrigation sequences (SI) using a Vickers microhardness test (VHN).

Methods: 20 samples were prepared by longitudinal cutting of 10 unirradiculated human tooth. Two IS were prepared, a traditional one $(5 \% \mathrm{NaOCl}$, saline $0,9 \%, 17 \%$ EDTA and saline $0,9 \%$ ) and a modified one (traditional plus a final irrigation of $5 \% \mathrm{NaOCl}$ ), each irrigant isolated in a glass plate. In two groups of 10 samples (traditional IS and modified IS) 5 identations were performed by a Vickers microdurometer prior and after the IS immersion of the samples. The average of Vickers microhardness (VMT) of the samples was obtained and compared statistically. $0.05 \mathrm{P}$ value.

Results: A statistical difference of superficial microhardness was found among pre-irrigation (control) and post-irrigation in both protocols. Nonetheless, there was not a statistical difference among both protocols. Use of EDTA followed by $\mathrm{NaOCl}$ did not show a significant decrease of dentinal superficial microhardness.

Conclusion: Use of $\mathrm{NaOCl}$ after EDTA did not show a significant decrease of dentinal superficial microhardness.

\section{INTRODUCCIÓN}

La desinfección de la mayor parte del sistema de conducto radicular (SCR) se logra con la acción de agentes irrigantes (AI). Estos facilitan la preparación mecánica del canal radicular principal, siendo su objetivo incrementar la probabilidad de éxito de un tratamiento endodóntico.

Un irrigante ampliamente utilizado es el Ácido etilendiaminotetracético (EDTA), agente ligeramente alcalino, que desmineraliza la dentina y remueve el barro dentinario. Por otra parte, el Hipoclorito de Sodio ( $\mathrm{NaOCl}$ ) se prefiere por su acción antimicrobiana y disolvente de tejido orgánico ${ }^{1}$. Debido a tales características, y como aún no se ha reportado un AI ideal que cumpla todos los requerimientos, se utilizan combinaciones de estos. $^{1}$

La erosión de la dentina por acción de AI expone tejido orgánico y resta contenido mineral, estos eventos tienen impacto en la microdureza superficial de la dentina (MSD), que ha sido una propiedad estudiada a través de ensayos de microdureza Vickers.

La secuencia de irrigación tradicional con $\mathrm{NaOCl}$ - Suero fisiológico (S) - EDTA y S nuevamente, tiene alta recomendación ${ }^{2}$, debido a que se combina la acción disolvente del $\mathrm{NaOCl}$ con el efecto quelante del EDTA. Se ha propuesto añadir $\mathrm{NaCl}$ al final de dicho procedimiento con el fin de potenciar su acción de disolvente orgánico y bactericida al abarcar niveles más profundos de la dentina ${ }^{2}$, sin embargo, esto provocaría una mayor erosión dentinaria, asociándose a una significativa disminución de la $\mathrm{MSD}^{3}$. Hasta el momento no se han encontrado estudios que evalúen este efecto comparando ambos métodos. Es por ello que consideramos pertinente preguntarse si existe una diferencia relevante en la MSD entre la secuencia de irrigación $\mathrm{NaOCl}$ - S - EDTA y $S$, y la modificación de ésta con un lavado final usando $\mathrm{NaOCl}$.

\section{MATERIALES Y MÉTODOS}

Diseño de estudio experimental in vitro de ciego simple, para comparar el efecto en la MSD las secuencias de irrigación tradicional $(\mathrm{NaOCl} 5 \%$, suero $0,9 \%$, EDTA $17 \%$ y suero $0,9 \%$ ) y modificada $(\mathrm{NaOCl} 5 \%$, suero $0,9 \%$, EDTA $17 \%$, suero $0,9 \%$ y $\mathrm{NaOCl} 5 \%$ ).

La muestra se conformó por la selección aleatoria de 10 dientes, de todos los extraídos en la Facultad de Odontología de la Universidad de Valparaíso por indicación terapéutica durante el año 2017. Los criterios de inclusión fueron diente permanente unirradiculado con indicación de extracción terapéutica, raíz indemne sin anomalías anatómicas, ápice cerrado, y como exclusión se consideró dientes con tratamiento endodóntico y fractura del diente durante la extracción.

Se eliminaron restos de tejido orgánico e inorgánico de la superficie radicular mediante uso de curetas e irrigación con suero y luego se conservaron en suero fisiológico al $0,9 \%$ y a 4 grados Celsius posterior a su extracción. 
Finalmente, fueron esterilizados con calor húmedo previo a realizar las pruebas de microdureza Vickers (VHN). Se calculó el tamaño muestral según el estudio de Patil CR y Uppin V (4), cuyo resultado entregó la necesidad de usar 10 dientes, ajustando las posibles pérdidas (2 en total) (Figura 1).

Para preparar las muestras, se sumergió la corona de los dientes hasta el límite amelo cementario (LAC) en acrílico de autocurado en recipiente de plástico, y se rotuló con números del 1 al 10 de forma arbitraria. Luego se hizo un corte longitudinal de la raíz utilizando la máquina de cortadora de precisión (IsoMet ${ }^{\circledR}$, Buehler Ltd, Lake Bluff, IL, EUA) con irrigación. Las muestras fueron decoronadas a nivel del LAC usando la misma cortadora de precisión a baja velocidad e irrigación Así, se obtuvieron 20 muestras en total (Figura 2).

Las muestras fueron montadas horizontalmente con acrílico de autocurado en anillos de cobre, exponiendo la zona interna de la raíz, y se

Figura 1: Diagrama Ilustrativo de la Metodología de Trabajo Realizada

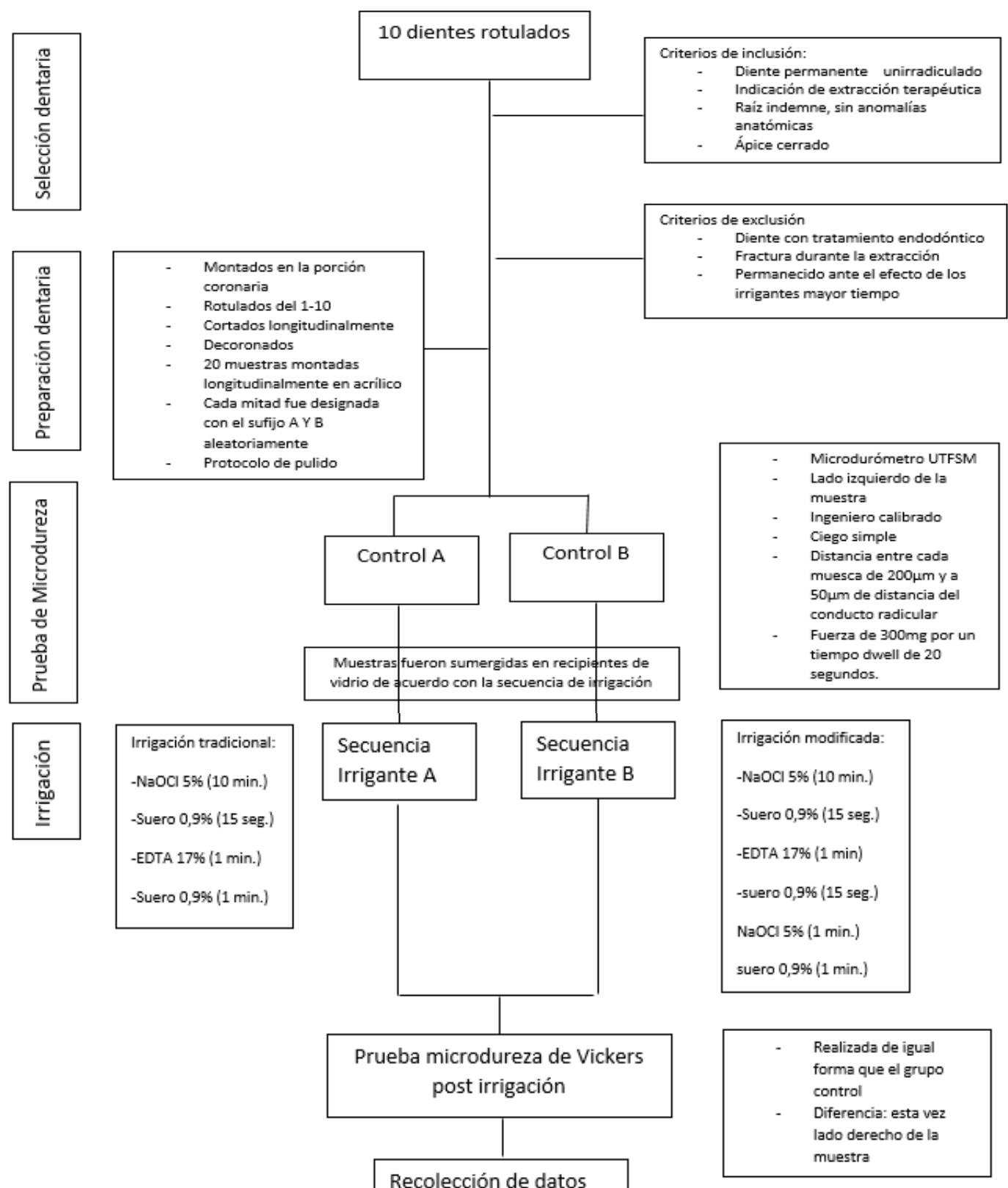


asignaron aleatoriamente 10 muestras para la secuencia tradicional y para la secuencia modificada y se rotuló nuevamente con números del 1 al 10 cada muestra luego de la asignación.

Figura 2: Corte longitudinal de un diente montado para la obtención de cuerpos de prueba

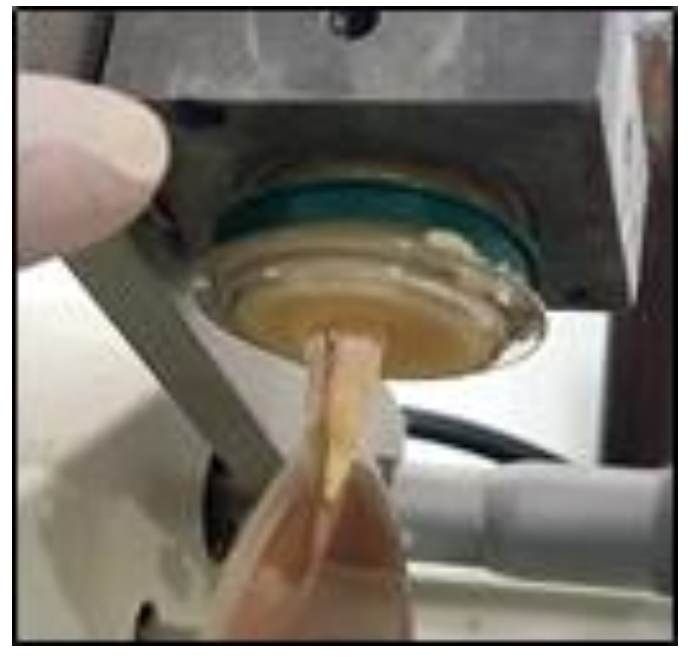

Figura 3: Imágenes ilustrativas de las identaciones de prueba de microdureza realizadas con microdurómetro Vickers a un aumento de 100x (imagen A) y 400x (imagen B)

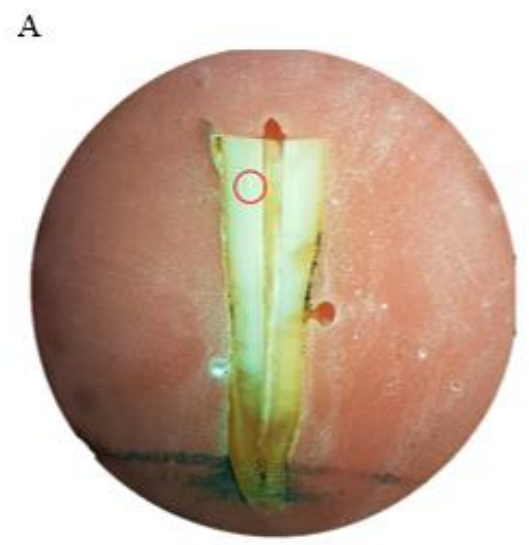

B

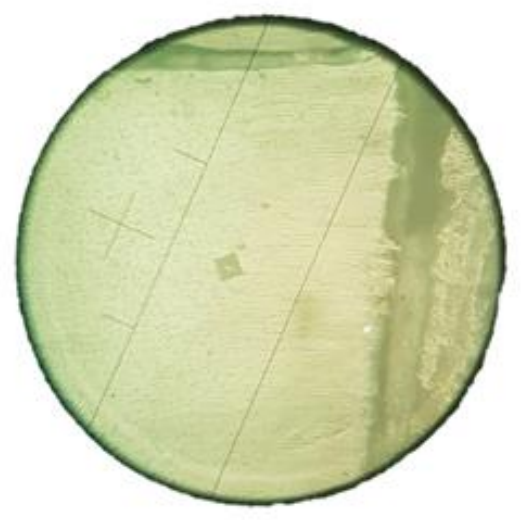

Se aplicó un protocolo de pulido para la superficie de las muestras con el sistema Sof-lex (3M ESPE, St Paul, MN, EUA), y aplicación de pasta de pulido diamantada prisma-gloss (Dentsply Caulk, Milford, DE, EUA) con irrigación de agua destilada.

Se realizó el test de microdureza de Vickers en cada muestra, previo a las secuencias de irrigación, con un microdurómetro Vickers de la Universidad Técnica Federico Santa María, (Valparaíso, Chile), con operador calibrado. Se realizaron identaciones a $200 \mu \mathrm{m}$ desde la porción coronal hasta $1 \mathrm{~mm}$ antes de la porción apical, con una distancia entre cada muesca de $200 \mu \mathrm{m}$ y a $50 \mu \mathrm{m}$ de distancia del conducto radicular (Figura 3). Esta medición se realizó a la izquierda del conducto radicular, aplicando una fuerza de $300 \mathrm{mg}$ por un tiempo dwell de 20 segundos. Así se obtienen las mediciones de control (C) para el grupo de muestras de la secuencia tradicional (CA) y secuencia modificada (CB), de 10 muestras cada uno.

Posterior a la prueba control, las muestras de cada grupo fueron sumergidas en las secuencias de irrigación asignadas (A y B), cada una contenidas en un recipiente de vidrio. El tiempo de exposición para el grupo de la secuencia tradicional, fue de 10 minutos en $\mathrm{NaOCl} 5 \%, 15$ segundos en SF 0.9\%, 1 minuto en EDTA al $17 \%$, y finalmente 1 minuto en SF $0.9 \%$. En el grupo de secuencia modificada se aplicó el mismo protocolo, al que se sumó una posterior exposición de 1 minuto en $\mathrm{NaOCl} 5 \%$, para terminar con 1 minuto de SF 0,9\%. Para evitar la contaminación de las soluciones irrigadoras, estas fueron cambiadas de los recipientes, entre una muestra y otra. Al final del procedimiento, las muestras fueron secadas con papel absorbente. Luego de ello, se realizó una prueba de microdureza Vickers (VHN) posterior a las secuencias de irrigación, con el mismo procedimiento, máquina, operador y lugar en el lado derecho de las muestras, obteniendo los valores de VHN para las muestras sumergidas en la secuencia tradicional (A) y modificada (B).

Los datos obtenidos fueron ingresados en el software Microsoft Excel 2013 ordenados del 1 al 10 para grupos $\mathrm{CA}, \mathrm{CB}, \mathrm{A}$ y $\mathrm{B}$ respectivamente. 
Se obtuvo los promedios de VHN por muestra en su fase de control, post irrigación, y se obtuvo el promedio entre la fase de control y post irrigación, registrándose como $\triangle \mathrm{A}(\mathrm{CA}-\mathrm{A})$ y $\Delta \mathrm{B}(\mathrm{CB}-\mathrm{B})$. Se realizaron pruebas estadísticas utilizando el software STATA versión 12.0, y se consensuó un p valor de 0,05 para establecer significancia estadística.

La variable estudiada es MSD, mediante el registro de microdureza Vickers. Se verificó la normalidad de sus datos con la prueba de Shapiro - Wilks, y la homogeneidad de varianzas se demostró con el test de Bartlett. Para comparación de promedios de VHN de los grupos control CA y $\mathrm{CB}$ se realizó el test $\mathrm{U}$ de Mann - Whitney.

El financiamiento de este estudio fue mediante el aporte directo de los investigadores, y se elaboró un consentimiento informado para que los pacientes atendidos por extracción dental en la Facultad de Odontología de la Universidad de Valparaíso donaran voluntariamente su diente para esta investigación, se prometió absoluta confidencialidad de datos personales. El manejo de residuos biológicos y desarrollo de la investigación contó con la aprobación del comité ético científico de la Universidad de Valparaíso.

La prueba de Shapiro - Wilks, que entregó $\mathrm{p}$ valores > 0,05 en todos los grupos, excepto en los valores de VHN previos a secuencia de irrigación tradicional (CA). La homogeneidad de varianzas se demostró con el test de Bartlett, dando $\mathrm{p}$ valores > 0,05 para la diferencia de VHN pre irrigación y post irrigación de la secuencia tradicional (CA-A) y la media entre la variación de $\mathrm{VHN}$ de las muestras asignadas a la secuencia tradicional $(\Delta \mathrm{A})$ y modificada $(\Delta \mathrm{B})$, excepto para la diferencia VHN pre irrigación $\mathrm{y}$ post irrigación de secuencia modificada (CB-B) que tuvo un $\mathrm{p}$ valor $<\mathrm{a} 0.05$. El valor mínimo de disminución se estableció en 6,33 VHN.

Se obtuvieron los promedios de dureza Vickers de las muestras (Figura 4). La prueba $U$ de Mann - Whitney $(\mathrm{p}=0.05)$ demostró $\mathrm{p}$ valores < 0,05 para las diferencias CA-A y CB-B, no así para las comparaciones de valores previos a la secuencia tradicional (CA) y modificada (CB), y tampoco para los valores post irrigación con secuencia tradicional y modificada (A y B; p > $0,05)$. La prueba $\mathrm{T}$ paramétrica independiente $(\mathrm{p}$ $=0,05)$ aplicada a los valores $\Delta \mathrm{A}$ y $\Delta \mathrm{B}$ tuvo un $\mathrm{p}$ valor > a 0.05 (Tabla I y II, Figura 4).

\section{DISCUSIÓN}

En nuestro estudio se encontraron diferencias estadísticamente significativas entre los promedios de VHN previos a las secuencias de irrigación respecto de las mediciones posteriores a ellas. Sin embargo, entre los valores promedio de microdureza Vickers de las mediciones posteriores a la exposición de las secuencias tradicional y modificada, y en la comparación de la variación de VHN entre secuencias de irrigación, no hubo diferencias estadísticamente significativas.

El $\mathrm{NaOCl}$ se utiliza en concentraciones de $0,5 \%$ a $6 \%$, y a pesar de tener un efecto de barrido de smear layer ${ }^{5}$, hay autores que han reportado una baja efectividad en este punto. ${ }^{6}$ La influencia en la ultraestructura dentinaria ${ }^{7}$ se da por la degradación de fibras colágenas. ${ }^{8,9}$ Esto altera propiedades mecánicas como módulo elástico, resistencia a la fractura y MSD. ${ }^{7,10}$ El EDTA usado en concentraciones de $15 \%$ o $17 \%$, ha demostrado ser efectivo en la remoción de barro dentinario $^{7,9}$ y la prevención de su formación. Al desmineralizar el tejido orgánico aumenta la permeabilidad dentinaria ${ }^{8}$, permitiendo el ingreso hacia sectores profundos del SCR y dentina de agentes desinfectantes y de medicación. ${ }^{2,11}$ El uso de suero fisiológico o agua destilada entre $\mathrm{NaOCl}$ y EDTA sirve para evitar que reaccionen entre ellos, pues el EDTA tiene un marcado efecto sobre la capacidad desinfectante del $\mathrm{NaOCl}^{2,11}$, no obstante, éste último debe aplicarse profusamente para remover el detrito que deja el EDTA, a lo que surge la propuesta de combinar estos AI para conseguir un efecto de irrigación ideal. $^{5,10,12,18}$

El nivel de afectación de MSD por AI ha descrito en varias investigaciones ${ }^{8}$ dependiendo de la concentración utilizada y del tiempo de exposición al $\mathrm{AI}^{12,13,14}$, y esto determina también 
Tabla I: Resultados por diferencias de VHN

\begin{tabular}{|c|c|c|c|c|c|c|c|c|}
\hline \multicolumn{4}{|c|}{$\begin{array}{l}\text { Normalidad y Homocedasticidad } \\
\text { de las variables }\end{array}$} & $\begin{array}{l}\text { Análisis descriptivo } \\
\text { de las variables }\end{array}$ & \multirow{2}{*}{\multicolumn{4}{|c|}{$\begin{array}{l}\text { Análisis estadístico } \\
\text { de los grupos estudiados }\end{array}$}} \\
\hline \multicolumn{2}{|c|}{ Grupos de estudio } & \multirow{2}{*}{$\begin{array}{c}\text { Test } \\
\begin{array}{c}\text { Shapiro -Wilks } \\
p=0.05\end{array} \\
0.34\end{array}$} & \multirow{3}{*}{$\begin{array}{c}\begin{array}{c}\text { Test } \\
\text { Bartlett } \\
p=0.05\end{array} \\
0.39\end{array}$} & $\begin{array}{l}\text { Promedio de } \\
\text { VHN por grupo }\end{array}$ & & & & \\
\hline Control & CA & & & 51.65 & \multicolumn{4}{|c|}{$\begin{array}{l}\text { Test U de Mann - Whitney } \\
\qquad p=0.05\end{array}$} \\
\hline Prueba & $\mathbf{A}$ & 0.68 & & 44.64 & $\mathbf{C A}-\mathbf{A}$ & 0.001 & CB - B & 0.01 \\
\hline Control & CB & 0.09 & \multirow{2}{*}{0.01} & 49.44 & \multicolumn{4}{|c|}{$\begin{array}{l}\text { Test Wilcoxon } \\
\quad p=0.05\end{array}$} \\
\hline Prueba & B & 0,08 & & 43.00 & $A-B$ & $>0,05$ & $\mathrm{CA}-\mathrm{CB}$ & 0.33 \\
\hline \multicolumn{4}{|c|}{ Promedio de VHN total } & 47.18 & & & & \\
\hline
\end{tabular}

Tabla II: Resultados por grupos de prueba

\begin{tabular}{|c|c|c|c|c|c|c|}
\hline \multicolumn{4}{|c|}{$\begin{array}{l}\text { Normalidad y Homocedasticidad } \\
\text { de las variables }\end{array}$} & $\begin{array}{l}\text { Análisis descriptivo } \\
\text { de las variables }\end{array}$ & \multirow{2}{*}{\multicolumn{2}{|c|}{$\begin{array}{l}\text { Análisis estadístico } \\
\text { de los grupos estudiados }\end{array}$}} \\
\hline \multicolumn{2}{|c|}{ Grupos de estudio } & $\begin{array}{c}\text { Test } \\
\text { Shapiro -Wilks } \\
p=0.05 \\
\end{array}$ & $\begin{array}{c}\text { Test } \\
\text { Bartlett } \\
p=0.05 \\
\end{array}$ & $\begin{array}{c}\text { Promedio de } \\
\text { diferencias de } \\
\text { VHN por grupo }\end{array}$ & & \\
\hline $\begin{array}{l}\text { Promedio } \\
\text { CA - A }\end{array}$ & $\Delta \mathrm{A}$ & $0, .42$ & \multirow{2}{*}{0.37} & 7.02 & \multicolumn{2}{|c|}{$\begin{array}{c}\text { Prueba T paramétrica } \\
\quad p=0.05\end{array}$} \\
\hline $\begin{array}{l}\text { Promedio } \\
\text { CB - B }\end{array}$ & $\Delta \mathrm{B}$ & 0.52 & & 6.44 & $\Delta \mathrm{A}-\Delta \mathrm{B}$ & 0.82 \\
\hline \multicolumn{4}{|c|}{ Promedio de diferencias de VHN total } & 5.78 & A - B & $p>0.05$ \\
\hline
\end{tabular}

Figura 4: Gráfico de distribución de los promedios de VHN de las muestras previo a las secuencias tradicional (CA), modificada (CB), y posterior a la exposición de las secuencias ( $A$ y $B$ ) respectivamente)

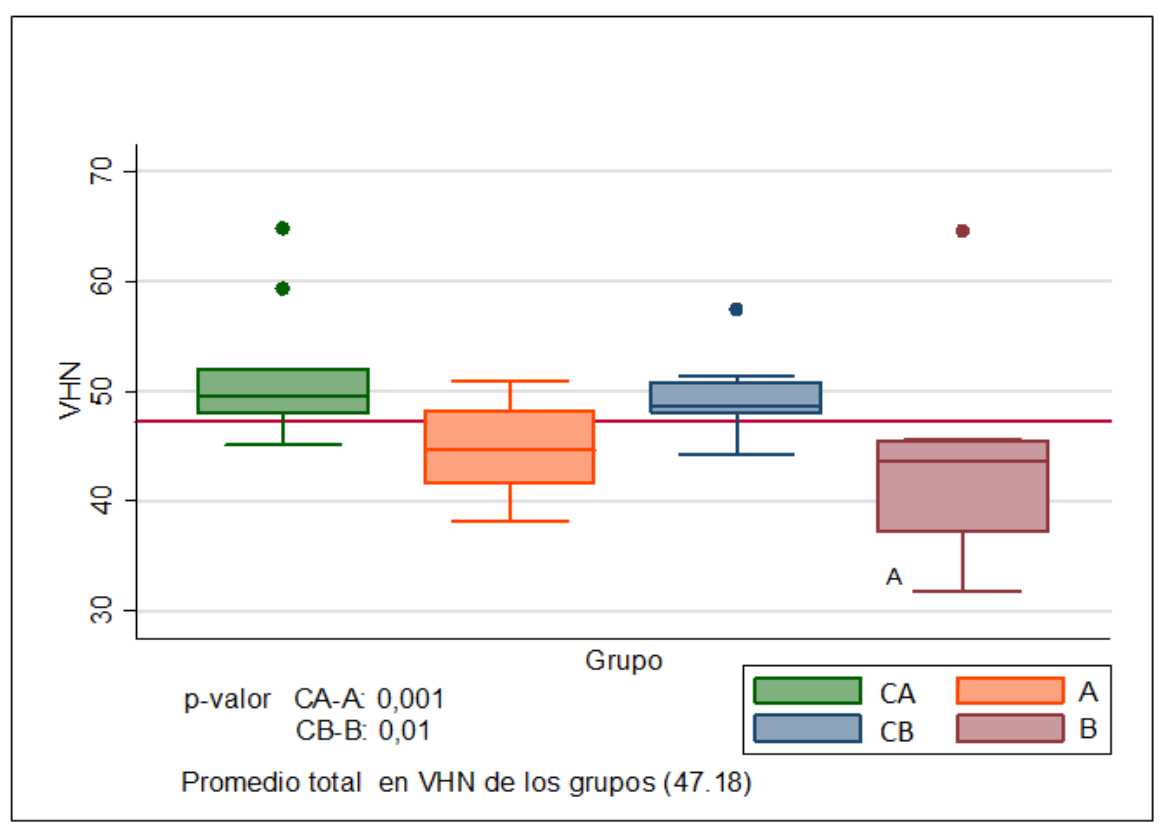


el alcance de penetración en el tejido. Se ha reportado que en concentraciones de $2 \%$ a $6 \%$ la penetración de $\mathrm{NaOCl}$ llega a 500 micrones, y esta investigación se utilizó $\mathrm{NaOCl}$ al $5 \%$ dado su mayor pote ncial bactericida ${ }^{5}$, pero no se midió la profundidad del efecto de las secuencias de irrigación, lo que sería suficiente y clínicamente relevante de investigar para estas secuencias de irrigación, pues la penetración de microorganismos alcanzaría 400 micrones de profundidad como máximo. ${ }^{15}$

Existe evidencia en la literatura que respalda los resultados obtenidos en este estudio en cuanto a comparación de promedios de VHN pre y post exposición a secuencias de irrigación de las muestras, reflejándose en una diferencia estadísticamente significativa de microdureza Vickers entre los dos momentos. Esto estaría explicado principalmente por la acción desmineralizante del EDTA, y existe evidencia que señala un marcado efecto de disminución de la microdureza superficial de la dentina al exponerla a este irrigante, lo que deja una estructura dependiente de tejido orgánico con propiedades mecánicas disminuidas. . $^{7,8}$

Uno de los factores que afecta la medición de la MSD, por medio de la de microdureza de Vickers, es la preparación de las muestras, ya que cualquier inclinación o superficie no plana, producirá una muesca demasiado grande y por tanto un valor de VHN menor o alterado. ${ }^{16}$ Por lo tanto, para producir una superficie plana en las muestras, se utilizó una secuencia de pulido específica, basada en estudios anteriores. ${ }^{4,17}$ Otros estudios han validado el uso de la máquina de microdureza de

Vickers para evaluar los cambios en las superficies dentales tratadas con agentes químicos. ${ }^{8,16}$

En este estudio, los tiempos de exposición de los irrigantes se definieron en base a literatura estudiada respecto al $\mathrm{NaOCl}$ y EDTA. Sin embargo, los hallazgos que fundamentan el uso de estos irrigantes se realizaron con tiempos de exposición diferentes, teniendo como máximo 5 minutos para una irrigación con $\mathrm{NaOCl}$, independiente de su concentración ${ }^{7,8,9}$, contrastando con los 10 minutos iniciales que utilizamos ya que sería tiempo suficiente para producir cambios estructurales en la dentina ${ }^{2}$, que es lo que buscábamos determinar. Esta situación no permite realizar una comparación con otros estudios, por lo que sería adecuado estudiar estas secuencias de irrigación con tiempos de exposición similares a los estudiados recientemente, los cuales pretenden acercarse a lo que ocurre en la práctica clínica.

La comparación de promedios de VHN posterior a las secuencias de irrigación tradicional y modificada no mostró diferencias estadísticamente significativas. Por otro lado, esta última permitirá una mayor penetración del $\mathrm{NaOCl}$ en los túbulos dentinarios y conductos asociados al canal radicular, lo que mejoraría la desinfección del $\mathrm{SCR}^{2,19}$, favorecería el pronóstico terapéutico del diente, además de acuerdo a la bibliografía consultada, el efecto en la MSD que genera el $\mathrm{NaOCl}$ es bajo comparado con el EDTA. ${ }^{8,9}$

\section{CONCLUSIÒN}

En este estudio encontramos una disminución estadísticamente significativa en los valores de microdureza superficial de la dentina tras realizarse ambas secuencias de irrigación endodóntica. Por otra parte no encontramos diferencias estadísticamente significativas entre los valores de ambas secuencias, lo que nos permite decir que la secuencia de irrigación modificada, que adiciona $\mathrm{NaOCl}$ y suero fisiológico posterior al uso de EDTA, produce una disminución en la microdureza superficial de la dentina similar a la secuencia tradicional.

\section{CONFLICTOS DE INTERÉS}

Sin conflictos de interés

\section{REFERENCIAS}

[1] Akcay I, Erdilek N, Bilge Hakan S. The efficacy of an experimental single solution versus alternate use of multiple irrigants on root dentin microhardness. Journal of Clinical 
and Experimental Dentistry. 2013;:e83-8.

[2] Haapasalo M, Shen Y, Wang Z, Gao Y. Irrigation in endodontics. British Dental Journal. 2014;216(6):299-303.

[3] Wei Qian, DDS, PhD, Ya Shen, DDS, PhD, Markus Haapasalo, DDS, PhD. Quantitative Analysis of the Effect of Irrigant Solution Sequences on Dentin Erosion.. 10, Vancouver : Journal of endodontics, 2011, Vol. 37, págs. 1437-1441.

[4] Patil Cuppin, V. Effect of endodontic irriganting solutions on the microhardness and roughness of root canal dentin: An in vitro study.. 22, 2011, Indian Journal of Dental Research, Vol. 1, pág. 22.

[5] Basrani B, Haapasalo M. Update on endodontic irrigating solutions. Endodontic Topics. 2012;27(1):74-102.

[6] Ulusoy Ö, Görgül G. Effects of different irrigation solutions on root dentine microhardness, smear layer removal and erosion. Australian Endodontic Journal. 2011;39(2):66-72.

[7] Ghisi A, Kopper P, Baldasso F, Stürmer C, Rossi-Fedele G, Steier L et al. Effect of Super-Oxidized Water, Sodium Hypochlorite and EDTA on Dentin Microhardness. Brazilian Dental Journal. 2014;25(5):420-424.

[8] Baldasso F, Roleto L, Silva V, Morgental R, Kopper P. Effect of final irrigation protocols on microhardness reduction and erosion of root canal dentin. Brazilian Oral Research. 2017;31(0).

[9] Saha S. Effectiveness of Various Endodontic Irrigants on the Micro-Hardness of the Root Canal Dentin: An in vitro Study. JOURNAL OF CLINICAL AND DIAGNOSTIC RESEARCH. 2017;

[10] Aaparolli, D., Saquy, P. and Cruz-Filho, A. (2012). Effect of sodium hypochlorite and edta irrigation, individually and in alternation, on dentin microhardness at the furcation area of mandibular molars. Brazilian Dental Journal, 23(6), pp.654-658.

[11] Azevedo R, Raquel A, Heberle A, Geraldo F, Karla de Pontes R, Dormelles, R. The effect of EDTA on the antibacterial activity of sodium hypochlorite. General Dentistry. 2018; January

2018:74-78.

[12] Zaparolli D, Saquy P, Cruz-Filho A. (2012). Effect of sodium hypochlorite and edta irrigation, individually and in alternation, on dentin microhardness at the furcation area of mandibular molars. Brazilian Dental Journal,
23(6), pp.654-658.

[13] Cobankara F, Erdogan H, Hamurcu M. (2011). Effects of chelating agents on the mineral content of root canal dentin. Oral Surgery, Oral Medicine, Oral Pathology, Oral Radiology, and Endodontology, 112(6), pp.e149-e154.

[14] Zhang K, Kim Y, Cadenaro M, Bryan T, Sidow S, Loushine R, et al. (2010). Effects of Different Exposure Times and Concentrations of Sodium Hypochlorite/Ethylenediaminetetraacetic Acid on the Structural Integrity of Mineralized Dentin. Journal of Endodontics, 36(1), pp.105109.

[15] Baldasso F, Roleto L, Silva V. Effect of final irrigation protocols on microhardness reduction and erosion of root canal dentin. Brasilia : Brazilian Oral Research, 2017.

[16] Saleh, H. A. J. Comparative evaluation of effect of irrigation solutions with various exposure time on microhardness of root canal dentin (In Vitro study). Irak :Iraqui Dental Journal, 2016.

[17] Gutiérrez-Salazar M, Reyes-Gasga J. Microhardness and chemical composition of human tooth. 3, México D.F. : Materials Research, 2003, Vol. 6.

[18] Kalluru R. (2014). Comparative Evaluation of the Effect of EDTA, EDTAC, $\mathrm{NaOCl}$ and MTAD on Microhardness of Human Dentin An In-vitro Study. JOURNAL OF CLINICAL AND DIAGNOSTIC RESEARCH.

[19] Haapasalo M, Shen Y, Quan W, Gao Y. 1, 2010, Dent Clin N Am, Vol. 54, págs. 291312.

\section{CÓMO CITAR ESTE ARTÍCULO}

Irigoyen A, Santis S, Soto I, Marchant C. Comparación in vitro del cambio de microdureza superficial dentinaria entre dos secuencias de irrigación endodóntica. Appli. Sci. Dent. 2020; 1(1): 1-8.

DOI: 10.22370/asd.2020.1.1.2107. 